\title{
Construction of Target Controllable Image Segmentation Model Based on Homotopy Perturbation Technology
}

\author{
Shu-Li Mei \\ College of Information and Electrical Engineering, China Agricultural University, Postbox 53, East Campus, \\ 17 Qinghua Donglu Road, Haidian District, Beijing 100083, China
}

Correspondence should be addressed to Shu-Li Mei; meishuli@163.com

Received 31 December 2012; Revised 4 January 2013; Accepted 6 January 2013

Academic Editor: Lan Xu

Copyright (C) 2013 Shu-Li Mei. This is an open access article distributed under the Creative Commons Attribution License, which permits unrestricted use, distribution, and reproduction in any medium, provided the original work is properly cited.

Based on the basic idea of the homotopy perturbation method which was proposed by Jihuan He, a target controllable image segmentation model and the corresponding multiscale wavelet numerical method are constructed. Using the novel model, we can get the only right object from the multiobject images, which is helpful to avoid the oversegmentation and insufficient segmentation. The solution of the variational model is the nonlinear PDEs deduced by the variational approach. So, the bottleneck of the variational model on image segmentation is the lower efficiency of the algorithm. Combining the multiscale wavelet interpolation operator and HPM, a semianalytical numerical method can be obtained, which can improve the computational efficiency and accuracy greatly. The numerical results on some images segmentation show that the novel model and the numerical method are effective and practical.

\section{Introduction}

In general, choosing different parameters in the most common image segmentation methods usually leads to different image segmentation results [1]. In other words, the object segmentation results are uncontrollable by the common methods. To solve the problem, one of the most common strategies is choosing thresholds using prior knowledge or analyzing the distribution of gray values of an image with the gray value histogram. Another method is image enhancement, which can often destroy the contour of the objects.

The variational method on image segmentation is a new image processing technology, which processes lots of better properties in processing medical images such as MRI and CI [2]. In this method, the pictures are taken as continuous energetic fields, and so the corresponding information in digital images such as gradient, divergence, and the curvature of the object contour can be viewed as the differential operators embedded in the variational model on image processing. The traditional complicated image processing such as denoising with texture preserving and exact segmentation can be done by this model. The outstanding work of this field is the energy function for image segmentation proposed by Mumford and
Shah, which has been widely used, and its mathematical properties are well analyzed. This is a general approach on image segmentation, where it is assumed that objects can be characterized by smooth surfaces or volumes in three dimensions. In order to solve the Mumford-Shah model with the Euler-Lagrange method, a simplified model was deduced by Chan and Vese, in which the Euclid length was employed instead of the Hausdorff length [3]. So, the simplified model is also called Chan-Vese model. Similar to other image segmentation methods, Chan-Vese model cannot identify the object as well. Multilevel set approach for solving C-V model can segment all the objects in a picture. But it will obviously lead to oversegmentation [4].

In many cases, the purpose of the image segmentation is to get one special single object instead of all the objects in a multiobject image. Therefore, the purpose of this paper is to construct a target controllable image segmentation model based on the basic idea of homotopy perturbation technology (HPM). Using the variational method, the optimal solution of the energy function can be expressed as a nonlinear partial differential equation. So, another task of this study is to construct an effective numerical method on nonlinear PDEs by combining the multiscale wavelet interpolation 

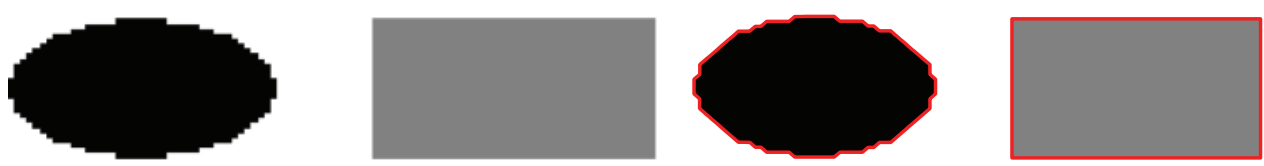

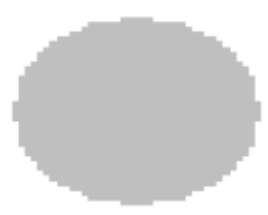

(a) Original image
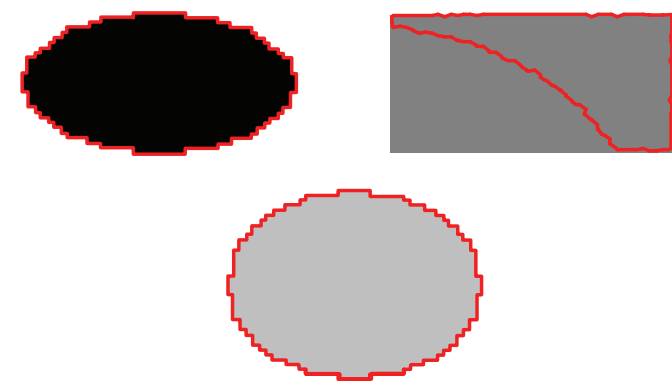

(c) 50 iteration times
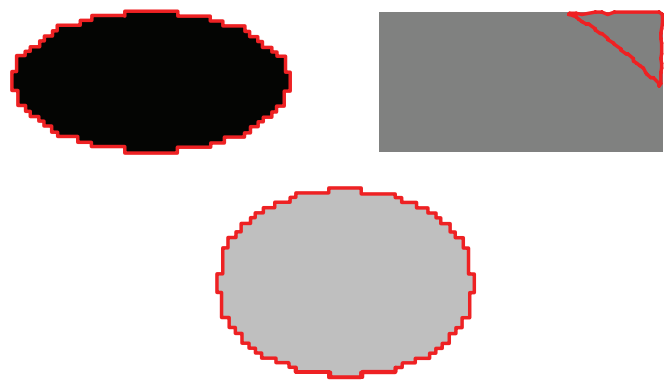

(e) 750 iteration times

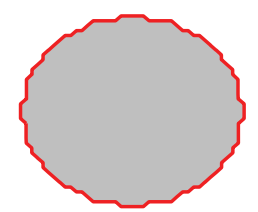

(b) 10 iteration times
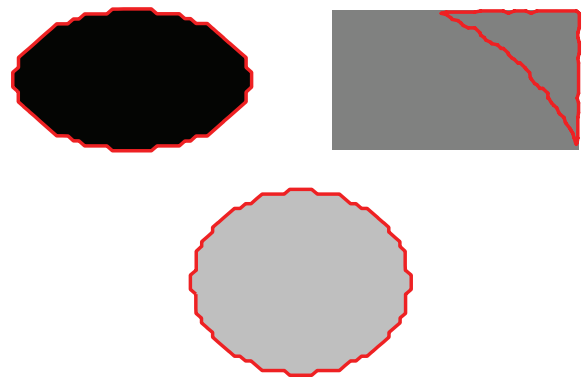

(d) 500 iteration times
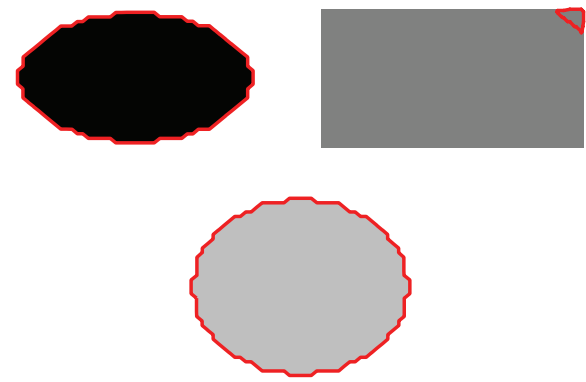

(f) 1000 iteration times

Figure 1: Multiobject image segmentation at $k=1\left(\lambda_{1}=\lambda_{2}=10, \Delta t=10\right)$.

operator and the homotopy perturbation method. The homotopy perturbation method (HPM) proposed by $\mathrm{He}[5,6]$ is constantly being developed and applied to solve various nonlinear problems by $\mathrm{He}$ [7-15] and by others [16-20]. The better improvement is adding an auxiliary parameter into the homotopy equation, which is helpful to eliminate the secular term in the perturbation solution. This can improve the rate of convergence greatly. Unlike analytical perturbation methods, HPM does not depend on small parameter which is difficult to find. The variational iteration method was another simple and effective method for nonlinear equations proposed by He [21-26], which can provide analytical approximations to a rather wide class of nonlinear equations [27-33] without linearization, perturbation, or discretization which can result in massive numerical computation. In order to solve the nonlinear PDEs, it is necessary to introduce the wavelet numerical algorithm [34-37] into HPM.

\section{Construction of Target Controllable Image Segmentation Model}

In order to solve the Mumford-Shah model with the EulerLagrange method, a simplified model was deduced by Chan and Vese, in which the Euclid length was employed instead of the Hausdorff length. This simplified model can also be called the Chan-Vese model, which can be expressed as follows:

$$
\begin{aligned}
& E^{\mathrm{CV}}\left(c_{1}, c_{2}, C\right)= \lambda_{1} \int_{\Omega_{1}}\left(I_{0}-c_{1}\right)^{2} d x d y \\
&+\lambda_{2} \int_{\Omega_{2}}\left(I_{0}-c_{2}\right)^{2} d x d y+v|C|, \\
& c_{i}=\operatorname{mean}_{\Omega_{i}}\left(u_{0}\right)=\frac{\int_{\Omega_{i}} u_{0}(x, y) d x d y}{\operatorname{Area}\left(\Omega_{i}\right)}, \quad i=1,2,
\end{aligned}
$$

where $\lambda_{1}$ and $\lambda_{2}$ are positive constants and $c_{1}$ and $c_{2}$ are the average gray level values inside $\left(\Omega_{1}\right)$ and outside $\left(\Omega_{2}\right)$ of the object contour, respectively. $I_{0}$ denotes the image to process, $|C|$ is the length of the object contour, and $v$ is the weight parameter. According to the level set method, the contour 
curves of the objects should be embedded into the level set function as follows:

$$
\begin{aligned}
C & =\{(x, y) \mid(x, y) \in \Omega, \phi(x, y)=0\}, \\
\Omega_{1} & =\{(x, y) \mid(x, y) \in \Omega, \phi(x, y)>0\}, \\
\Omega_{2} & =\{(x, y) \mid(x, y) \in \Omega, \phi(x, y)<0\} .
\end{aligned}
$$

Then, the level set-based $\mathrm{C}-\mathrm{V}$ model can be rewritten as follows:

$$
\begin{aligned}
& E\left(c_{1}, c_{2}, \phi\right)=\lambda_{1} \int_{\Omega}\left|I_{0}-c_{1}\right|^{2} H(\phi) d x d y \\
& +\lambda_{2} \int_{\Omega}\left|I_{0}-c_{2}\right|^{2}(1-H(\phi)) d x d y \\
& +v \int_{\Omega}|H(\phi)| d x d y \\
& H(\phi)=\left\{\begin{array}{ll}
1, & \phi \geq 0, \\
0, & \phi<0,
\end{array} \quad \delta_{\varepsilon}=\frac{\varepsilon}{\pi\left(\varepsilon^{2}+\phi^{2}\right)}\right.
\end{aligned}
$$

Using the variational method, the PDEs with respect to the variable $\phi$ can be obtained as follows:

$$
\frac{\partial \phi}{\partial t}=\delta_{\varepsilon}(\phi)\left[v \operatorname{div}\left(\frac{\nabla \phi}{|\nabla \phi|}\right)-\lambda_{1}\left|I_{0}-c_{1}\right|^{2}+\lambda_{2}\left|I_{0}-c_{2}\right|^{2}\right]
$$

Obviously, $\operatorname{div}(\nabla \phi /|\nabla \phi|)$ is the curvature of the level set function $\phi$, and $\delta_{\varepsilon}(\phi)$ is used to constrain the growth of the level set function.

The solution of (4) is the level set function $\phi(x, y, t)$ at time $t$. The zero level set is the object contour curve, which can be obtained by solving $\phi(x, y, t)=0$.

In the following, what we are talking about is how to construct the target controllable image segmentation model based on the basic idea of HPM. It is easy to understand that the function of the curvature in $\mathrm{C}-\mathrm{V}$ model is just to preserve the smoothness of the object contour. Neglecting the curvature in (4), the simplified model can be obtained as follows:

$$
\frac{\partial \phi}{\partial t}=\delta_{\varepsilon}(\phi)\left[-\lambda_{1}\left|I_{0}-c_{1}\right|^{2}+\lambda_{2}\left|I_{0}-c_{2}\right|^{2}\right]
$$

In solving the $\mathrm{C}-\mathrm{V}$ model with HPM and iteration method, the average gray level values inside and outside of the contour curves $c_{1}$ and $c_{2}$ vary with the evolution of the level set function. This evolution will end up with that the contour curve coincides with the object boundary. Then, $c_{1}$ and $c_{2}$ become constants, and the right hand of (5) should equal zero; that is,

$$
\frac{\lambda_{1}}{\lambda_{2}}=\frac{\left|I_{0}-c_{2}\right|^{2}}{\left|I_{0}-c_{1}\right|^{2}}
$$

In general, $\lambda_{1}$ and $\lambda_{2}$ are constant, which are correlated to $c_{1}$ and $c_{2}$ obviously.
It is easy to understand that the segmentation results and the values of $c_{1} / c_{2}$ are in one-to-one correspondence with each other. So, the object segmentation can be controlled by the value of $\left(\lambda_{1} / \lambda_{2}\right)$. Let $k=\lambda_{1} / \lambda_{2}, m=c_{1} / c_{2}$, and substitute $k, m$ into (5), we can obtain

$$
\frac{\partial \phi}{\partial t}=\delta_{\varepsilon}(\phi)\left[(1-k) I_{0}^{2}-2 I_{0} c_{2}(1-k m)+c_{2}^{2}\left(1-k m^{2}\right)\right]
$$

Let

$$
F\left(I_{0}\right)=(1-k) I_{0}^{2}-2 I_{0} c_{2}(1-k m)+c_{2}^{2}\left(1-k m^{2}\right)
$$

It is obviously that $F\left(I_{0}\right)=0$ is the necessary condition for the functional extremum problem about $\mathrm{C}-\mathrm{V}$ model. The solution of the necessary condition is

$$
\begin{aligned}
I_{0} & =\frac{2 c_{2}(1-k m) \pm \sqrt{4 c_{2}^{2}(1-k m)^{2}-4(1-k) c_{2}^{2}\left(1-k m^{2}\right)}}{2(1-k)} \\
& =\frac{\left(c_{2}-k c_{1}\right) \pm\left(c_{2}-c_{1}\right) \sqrt{k}}{1-k} .
\end{aligned}
$$

In the end of the image segmentation processing, the gray level value $I_{0}$ of the pixel inside the object contour equals $c_{2}$; that is, $I_{0}=c_{2}$ (in $\Omega_{2}$ ). Then, the relation between the parameter $k$ and the average gray level value of the image can be expressed as

$$
k=\left(\frac{c_{1}}{c_{2}-c_{1}}\right)^{2} \quad \text { or } \quad k=\left(\frac{2 c_{2}-c_{1}}{c_{2}-c_{1}}\right)^{2} .
$$

In the end of the image segmentation procedure, the final $c_{1}$ and $c_{2}$ should be coincident with average gray level values inside and outside of the segmentation target, respectively. They can be determined in advance by the priori knowledge. But in the beginning of the image segmentation processing, $c_{1}$ and $c_{2}$ are the average gray level values inside and outside of the zero level set, respectively. They are determined by the position of the level set function, which is random in most cases. It is easy to understand that there is a continuous map between the two cases, that is, the connection between the two cases can be set up by the HPM. In other words, the parameter $k$ in (10) can be taken as the homotopy parameter; then, a linear homotopy function for (4) can be constructed as

$$
\begin{aligned}
& \frac{\partial \phi}{\partial t}-\delta_{\varepsilon}(\phi)\left[v \operatorname{div}\left(\frac{\nabla \phi}{|\nabla \phi|}\right)-\left|I_{0}-c_{1}\right|^{2}\right]+p \delta_{\varepsilon}(\phi)\left|I_{0}-c_{2}\right|^{2} \\
& +\alpha p(1-p) \phi=0,
\end{aligned}
$$



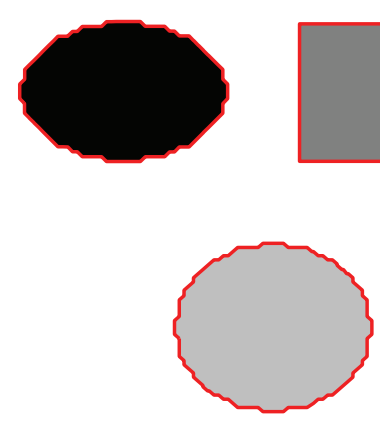

(a) 10 iteration times
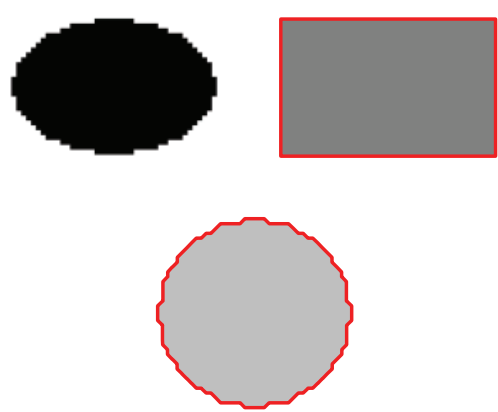

(b) 1000 iteration times

FIGURE 2: Multitarget image segmentation with target controllable model.

where the homotopy parameter $p \in[1, k], k$ is determined by the final $c_{1}$ and $c_{2}$ based on the priori knowledge in advance and $v$ is a weight parameter. $\alpha$ is an auxiliary parameter, which can be identified by eliminating the secular term in the perturbation analytical solution.

Equation (11) is the target controllable image segmentation model. It should be pointed out that the auxiliary parameter $\alpha$ appearing in this model is set to zero, as there is no any secular term in the perturbation analytical solution.

\section{HPM on Nonlinear System Based on the Multilevel Wavelet Analysis}

3.1. Wavelet Numerical Discretization Schemes on C-V Model. The definition domain of the image is defined as $\left(x_{\min }, x_{\max }\right) \times$ $\left(y_{\min }, y_{\max }\right)$, which should be divided evenly into $2^{j} \times 2^{j}(j$ is the level number) subdomains according to the wavelet collocation method. The connection nodes between two adjoining subdomains are the discretization points defined as $\left(x_{k_{1}}^{j}, y_{k_{2}}^{j}\right)$, where

$$
\begin{aligned}
& x_{k_{1}}^{j}=x_{\min }+k_{1} \frac{x_{\max }-x_{\min }}{2^{j}}, \\
& y_{k_{1}}^{j}=y_{\min }+k_{2} \frac{y_{\max }-y_{\min }}{2^{j}} .
\end{aligned}
$$

In addition, $w_{k 1, k 2}^{j(m, n)}(x, y)$ denotes the multiscale wavelet function and the corresponding $m$ th and $n$th derivatives with respect to $x$ and $y$, respectively. The level set function $\phi(x, y, t)$ and the corresponding derivative function can be descretized as follows:

$$
\begin{array}{r}
\phi^{J(m, n)}(x, y, t) \\
=\sum_{k_{01}=0}^{1} \sum_{k_{02}=0}^{1} \phi\left(x_{k_{01}}^{0}, y_{k_{02}}^{0}\right) w_{k_{01}, k_{02}}^{0(m, n)}(x, y) \\
+\sum_{j=0}^{J-1} \sum_{k_{11}=0}^{2^{j}-1} \sum_{k_{12}=0}^{2^{j}-1}\left[\alpha_{j, k_{11}, k_{12}}^{1}(t) w_{2 k_{11}+1,2 k_{12}}^{j+1(m, n)}(x, y)\right. \\
+\alpha_{j, k_{11}, k_{12}}^{2}(t) w_{2 k_{11}, 2 k_{12}+1}^{j+1(m, n)}(x, y) \\
\left.+\alpha_{j, k_{11}, k_{12}}^{3}(t) w_{2 k_{11}+1,2 k_{12}+1}^{j+1(m, n)}(x, y)\right],
\end{array}
$$

where $j$ and $J$ are constants, which denote the wavelet scale number and the maximum of the scale number, respectively. $\alpha_{j, k_{11}, k_{12}}^{1}, \alpha_{j, k_{11}, k_{12}}^{2}$, and $\alpha_{j, k_{11}, k_{12}}^{3}$ are the wavelet coefficients at the discretization point $\left(x_{k_{1}}^{j}, y_{k_{2}}^{j}\right)$.

According to above definitions, the curvature of the level set $\phi(x, y, t)$ can be expressed approximately as

$$
\begin{aligned}
\operatorname{div}\left(\frac{\nabla \phi}{|\nabla \phi|}\right) \approx & \operatorname{div}\left(\frac{\nabla \phi^{J}}{\left|\nabla \phi^{J}\right|}\right) \phi^{J(2,0)}(x, y, t)\left(\phi^{J(0,1)}(x, y, t)\right)^{2} \\
+ & \phi^{J(0,2)}(x, y, t)\left(\phi^{J(1,0)}(x, y, t)\right)^{2} \\
= & \left(-2 \phi^{J(0,1)}(x, y, t) \phi^{J(1,0)}(x, y, t) \phi^{J(1,1)}\right. \\
& \times(x, y, t)) \\
\times & \left(\left(\phi^{J(0,1)}(x, y, t)\right)^{2}\right. \\
& \left.+\left(\phi^{J(1,0)}(x, y, t)\right)^{2}\right)^{-3 / 2} .
\end{aligned}
$$

Substituting (14) and (13) into (11), we obtain

$$
\begin{aligned}
& \frac{\partial \phi^{J}(x, y, t)}{\partial t}-\delta_{\varepsilon}\left(\phi^{J}(x, y, t)\right) \\
& \quad \times\left[v \operatorname{div}\left(\frac{\nabla \phi^{J}(x, y, t)}{\left|\nabla \phi^{J}(x, y, t)\right|}\right)-\left|I_{0}-c_{1}\right|^{2}\right] \\
& \quad+p \delta_{\varepsilon}\left(\phi^{J}(x, y, t)\right)\left|I_{0}-c_{2}\right|^{2}=0 .
\end{aligned}
$$

Obviously, (15) is a nonlinear ordinary differential equation. 


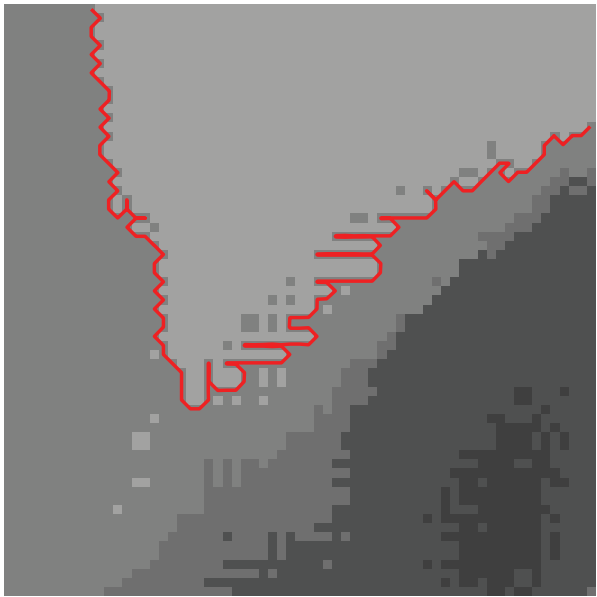

(a) C-V model

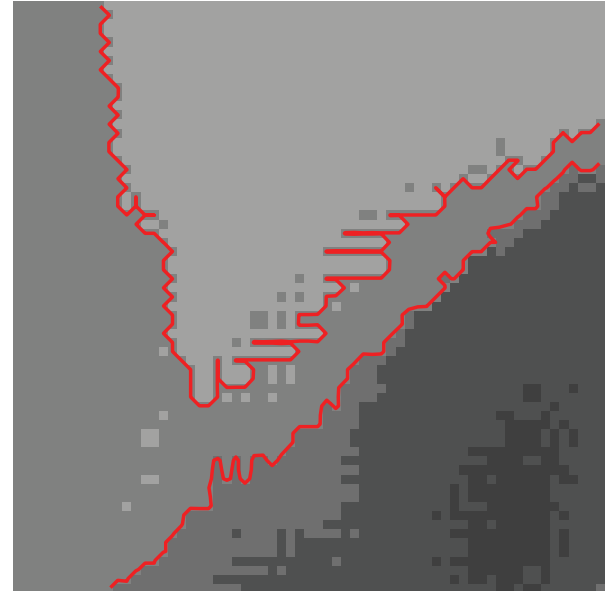

(b) Target controllable model

FIGURE 3: Special object segmentation.

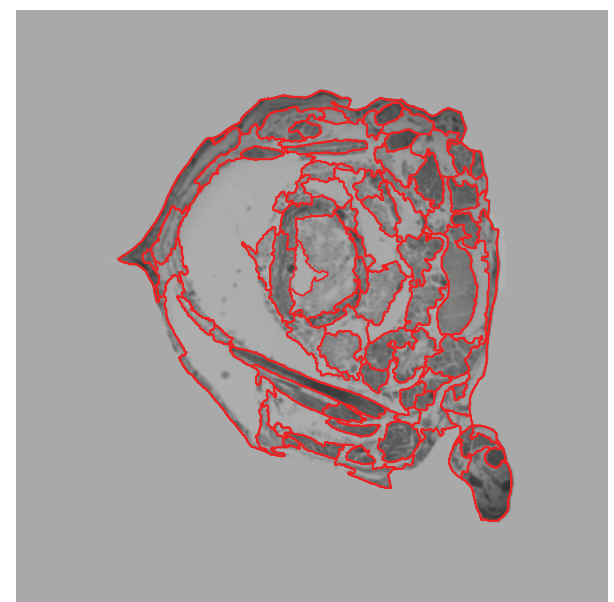

FIGURE 4: Locust's coelom image segmentation results by the target controllable model.

3.2. HPM on Discretization Format of C-V Model. There are various ways to construct a homotopy function. For (15), a linear homotopy function can be constructed as

$$
\frac{d \phi^{J}(x, y, t)}{d t}=(1-\varepsilon) F_{n}+\varepsilon F_{n+1}, \quad t \in\left[t_{n}, t_{n+1}\right]
$$

where

$$
\begin{aligned}
F_{n}=\delta_{\varepsilon}\left(\phi^{J}\left(x, y, t_{n}\right)\right)[ & v \operatorname{div}\left(\frac{\nabla \phi^{J}\left(x, y, t_{n}\right)}{\left|\nabla \phi^{J}\left(x, y, t_{n}\right)\right|}\right)-\left|I_{0}-c_{1}\right|^{2} \\
& \left.+p\left|I_{0}-c_{2}\right|^{2}\right], \quad n \in \mathbb{Z},
\end{aligned}
$$

where $v$ is the weight parameter. According to the perturbation theory, the solution of (16) can be expressed as the power series expansion of $p$

$$
\phi^{J}(x, y, t)=\phi_{0}^{J}(x, y, t)+\varepsilon \phi_{1}^{J}(x, y, t)+\varepsilon^{2} \phi_{2}^{J}(x, y, t)+\cdots .
$$

Substituting (18) into (16) and rearranging based on powers of $\varepsilon$-terms, we have

$$
\begin{aligned}
& \varepsilon^{0}: \frac{d \phi_{0}^{J}(x, y, t)}{d t}=F_{n} \\
& \varepsilon^{1}: \frac{d \phi_{1}^{J}(x, y, t)}{d t}=f_{1} \\
& \varepsilon^{2}: \frac{d \phi_{2}^{J}(x, y, t)}{d t}=f_{2}
\end{aligned}
$$

where $f_{1}$ and $f_{2}$ are functions with respect to $\varepsilon^{1}$ and $\varepsilon^{2}$, respectively. It is easy to identify the homotopy parameter as

$$
\varepsilon(t)=\frac{t-t_{n}}{t_{n+1}-t_{n}}, \quad t \in\left[t_{n}, t_{n+1}\right] .
$$

Thus, based on the definition of the Taylor series, $\phi_{1}^{J}(x, y, t)$ can be identified as $\left(t_{n+1}-t_{n}\right) F_{n}$, and $f_{1}$ can be identified as

$$
\begin{aligned}
f_{1}=\left(t_{n+1}-t_{n}\right) & \\
\times \frac{d}{d t}\left\{\delta_{\varepsilon}\left(\phi^{J}(x, y, t)\right)\right. & {\left[v \operatorname{div}\left(\frac{\nabla \phi^{J}(x, y, t)}{\left|\nabla \phi^{J}(x, y, t)\right|}\right)\right.} \\
& \left.\left.-\left|I_{0}-c_{1}\right|^{2}+p\left|I_{0}-c_{2}\right|^{2}\right]\right\} .
\end{aligned}
$$


Substituting $\phi_{0}^{J}(x, y, t)$ and $\phi_{1}^{J}(x, y, t)$ into (18) and assuming $\varepsilon=1$, the numerical solution of (16) can be obtained subsequently:

$$
\phi^{J}\left(x, y, t_{n+1}\right)=\phi_{0}^{J}\left(x, y, t_{n}\right)+\phi_{1}^{J}\left(x, y, t_{n}\right) .
$$

Then, the wavelet coefficient can be obtained as follows:

$$
\begin{aligned}
& \alpha_{j, k 1, k 2}^{1}\left(t_{n+1}\right)= \phi^{J}\left(x_{2 k 1+1}^{j+1}, y_{2 k 2}^{j+1}, t_{n+1}\right) \\
&- {\left[\sum_{k 01=0}^{1} \sum_{k 02=0}^{1} \phi^{J}\left(x_{k 01}^{0}, y_{k 02}^{0}, t_{n+1}\right)\right.} \\
& \times w_{k 01, k 02}^{0}\left(x_{2 k 1+1}^{j+1}, y_{2 k 2}^{j+1}\right) \\
&+\sum_{j 1=0}^{j-1} \sum_{k 11=0}^{2^{j 1}} \sum_{k 12=0}^{2^{j 2}}\left(\alpha_{j 1, k 11, k 12}^{1} w_{2 k 11+1,2 k 12}^{j 1+1}\right. \\
& \times\left(x_{2 k 1+1}^{j+1}, y_{2 k 2}^{j+1}\right) \\
&+\alpha_{j 1, k 11, k 12}^{2} w_{2 k 11,2 k 12+1}^{j 1+1} \\
& \times\left(x_{2 k 1+1}^{j+1}, y_{2 k 2}^{j+1}\right) \\
&+\alpha_{j 1, k 11, k 12}^{3} \\
& \times w_{2 k 11+1,2 k 12+1}^{j 1+1} \\
&\left.\left.\times\left(x_{2 k 1+1}^{j+1}, y_{2 k 2}^{j+1}\right)\right)\right]
\end{aligned}
$$$$
\alpha_{j, k 1, k 2}^{2}\left(t_{n+1}\right)=\phi^{J}\left(x_{2 k 1}^{j+1}, y_{2 k 2+1}^{j+1}, t_{n+1}\right)
$$$$
-\left[\sum_{k 01=0}^{1} \sum_{k 02=0}^{1} \phi^{J}\left(x_{k 01}^{0}, y_{k 02}^{0}, t_{n+1}\right)\right.
$$$$
\times w_{k 01, k 02}^{0}\left(x_{2 k 1}^{j+1}, y_{2 k 2+1}^{j+1}\right)
$$$$
+\sum_{j 1=0}^{j-1} \sum_{k 11=0}^{2^{j 1}} \sum_{k 12=0}^{2^{j 2}}\left(\alpha_{j 1, k 11, k 12}^{1} w_{2 k 11+1,2 k 12}^{j 1+1}\right.
$$$$
\times\left(x_{2 k 1}^{j+1}, y_{2 k 2+1}^{j+1}\right)
$$$$
+\alpha_{j 1, k 11, k 12}^{2} w_{2 k 11,2 k 12+1}^{j 1+1}
$$$$
\times\left(x_{2 k 1}^{j+1}, y_{2 k 2+1}^{j+1}\right)
$$$$
+\alpha_{j 1, k 11, k 12}^{3}
$$$$
\times w_{2 k 11+1,2}^{j 1+1}
$$$$
\left.\left.\times\left(x_{2 k 1}^{j+1}, y_{2 k 2+1}^{j+1}\right)\right)\right] \text {, }
$$

$$
\begin{aligned}
& \alpha_{j, k 1, k 2}^{3}\left(t_{n+1}\right)=\phi^{J}\left(x_{2 k 1+1}^{j+1}, y_{2 k 2+1}^{j+1}, t_{n+1}\right) \\
&-\left[\sum_{k 01=0}^{1} \sum_{k 02=0}^{1} \phi^{J}\left(x_{k 01}^{0}, y_{k 02}^{0}, t_{n+1}\right)\right. \\
& \times w_{k 01, k 02}^{0}\left(x_{2 k 1+1}^{j+1}, y_{2 k 2+1}^{j+1}\right) \\
&+\sum_{j 1=0}^{j-1} \sum_{k 11=0}^{2^{j 1}} \sum_{k 12=0}^{2^{j 2}}\left(\alpha_{j 1, k 11, k 12}^{1} w_{2 k 11+1,2 k 12}^{j 1+1}\right. \\
& \\
& \quad \times\left(x_{2 k 1+1}^{j+1}, y_{2 k 2+1}^{j+1}\right) \\
&+\alpha_{j 1, k 11, k 12}^{2} w_{2 k 11,2 k 12+1}^{j 1+1} \\
& \times\left(x_{2 k 1+1}^{j+1}, y_{2 k 2+1}^{j+1}\right) \\
&+\alpha_{j 1, k 11, k 12}^{3} \\
& \times w_{2 k 11+1,2 k 12+1}^{j 1+1} \\
&
\end{aligned}
$$

where

$$
x_{k}^{j}=x_{\min }+\frac{x_{\max }-x_{\min }}{2^{j}}, \quad y_{k}^{j}=y_{\min }+\frac{y_{\max }-y_{\min }}{2^{j}} .
$$

$w_{k 1, k 2}^{j}(x, y)$ is the quasi-Shannon wavelet function; that is,

$$
\begin{aligned}
& w_{k 1, k 2}^{j}(x, y)= \frac{\sin \left[\left(\pi / \Delta j_{1}\right)\left(x-x_{k 1}^{j}\right)\right]}{\left(\pi / \Delta \Delta j_{1}\right)\left(x-x_{k 1}^{j}\right)} \\
& \times \frac{\sin \left[\left(\pi / \Delta j_{2}\right)\left(y-y_{k 2}^{j}\right)\right]}{\left(\pi / \Delta j_{2}\right)\left(y-y_{k 2}^{j}\right)} \\
& \times \exp \left(-\frac{1}{2 r^{2}} \frac{\left(x-x_{k 1}^{j}\right)^{2}}{\left(\Delta j_{1}\right)^{2}}\right) \\
& \times \exp \left(-\frac{1}{2 r^{2}} \frac{\left(y-y_{k 2}^{j}\right)^{2}}{\left(\Delta j_{2}\right)^{2}}\right), \\
& \Delta j_{1}=\frac{x_{\max }-x_{\min }}{2^{j}}, \quad \Delta j_{2}=\frac{y_{\max }-y_{\min }}{2^{j}} .
\end{aligned}
$$


Substituting the three wavelet coefficients into (16), $\phi^{J(m, n)}\left(x, y, t_{n+1}\right)$ can be obtained; then we can obtain $F_{n+1}$ as follows:

$$
\begin{aligned}
F_{n+1}=\delta_{\varepsilon} & \left(\phi^{J}\left(x, y, t_{n+1}\right)\right) \\
\times & {\left[v \operatorname{div}\left(\frac{\nabla \phi^{J}\left(x, y, t_{n+1}\right)}{\left|\nabla \phi^{J}\left(x, y, t_{n+1}\right)\right|}\right)-\left|I_{0}-c_{1}\right|^{2}\right.} \\
& \left.+p\left|I_{0}-c_{2}\right|^{2}\right], \quad n \in \mathbb{Z} .
\end{aligned}
$$

At last, we can obtain the image segmentation result expressed in the level set as follows:

$$
\phi^{J}\left(x, y, t_{n+1}\right)=\phi^{J}\left(x, y, t_{n}\right)+\frac{t_{n+1}-t_{n}}{2}\left(F_{n}+F_{n+1}\right) .
$$

\section{Numerical Experiences and Discussion}

In this section, we take some multiobject images as examples to illustrate the efficiency of the target controllable image segmentation model compared with the $\mathrm{C}-\mathrm{V}$ model. The original image showed in Figure 1 consisted of three geometrical solid objects. The color of the background is white, and whole area is 1 . The gray level values and the areas of the three objects are showed in Table 1 . The image segmentation aims to get the circular and the rectangular objects. In other words, we want to take the black elliptical object as the background.

The segmentation results of $\mathrm{C}-\mathrm{V}$ model are showed in Figure 1. With the increasing of the iteration times, the rectangular object becomes a part of the background gradually instead of the elliptical object, which does not meet our requirement obviously.

The segmentation results with the target controllable model are showed in Figure 2 . The final $c_{1}=255 \times 0.7976 /$ $(0.7976+0.0568)=238.05$, and $c_{2}=(163 \times 0.0723+7 \times$ $0.0733) /(0.0723+0.0733)=86.464$. It should be pointed out that the final $c_{1}$ and $c_{2}$ can be obtained by priori knowledge in most cases. At the beginning of the segmentation, all the three objects are obtained as the foreground. With the increasing of the iteration times, the black object is gradually pushed into the background and out of the object region.

Figure 3 is an enlarged local image of the locust body cavity. The objects have an irregular shape with a slightly serrated border which can introduce over an insufficient segmentation. So it is difficult to segment with other methods. Using the target controllable model, we can get the right object easily. This example shows that the novel model and the corresponding numerical method are practical. Indeed, the novel model has been used to segment the locust's coelom images (Figure 4).

\section{Conclusions}

$\mathrm{C}-\mathrm{V}$ model is a kind of the modified Mumford-Shah model which has been widely used in medical images, and its
TABLE 1: The gray level values and areas of all objects.

\begin{tabular}{lcccc}
\hline & Ellipse & Circle & Squareness & Background \\
\hline Gray level value & 0 & 163 & 7 & 255 \\
Area & 0.0568 & 0.0723 & 0.0733 & 0.7976 \\
\hline
\end{tabular}

mathematical properties are well analyzed. But the segmentation result is usually uncontrollable. The target controllable image segmentation model proposed in this paper is based on the idea of HPM. The numerical experiences show that the novel model and the corresponding numerical algorithm are effective and practical. It meets the requirement of the medical images segmentation.

\section{Acknowledgment}

This work is supported by the National Natural Science Foundation of China under Grant no. 41171337 and National Key Technologies R \& D Program of China under Grant no. 2012BAD35B02.

\section{References}

[1] O. Wirjadi, "Survey of 3rd image segmentation methods," Berichte Des Fraunhofer ITWM 123, 2007.

[2] L. A. Vese and T. F. Chan, "A multiphase level set framework for image segmentation using the Mumford and Shah model," International Journal of Computer Vision, vol. 50, no. 3, pp. 271293, 2002.

[3] T. F. Chan, S. Esedoglu, and M. Nikolova, "Algorithms for finding global minimizers of image segmentation and denoising models," UCLA Report, 2004.

[4] T. Goldstein, X. Bresson, and S. Osher, "Geometric applications of the split Bregman method: segmentation and surface reconstruction," Journal of Scientific Computing, vol. 45, no. 1-3, pp. 272-293, 2010.

[5] J.-H. He, "A coupling method of a homotopy technique and a perturbation technique for non-linear problems," International Journal of Non-Linear Mechanics, vol. 35, no. 1, pp. 37-43, 2000.

[6] J.-H. He, "Homotopy perturbation technique," Computer Methods in Applied Mechanics and Engineering, vol. 178, no. 3-4, pp. 257-262, 1999.

[7] J.-H. He, "Asymptotology by homotopy perturbation method," Applied Mathematics and Computation, vol. 156, no. 3, pp. 591596, 2004.

[8] J.-H. He, "Homotopy perturbation method: a new nonlinear analytical technique," Applied Mathematics and Computation, vol. 135, no. 1, pp. 73-79, 2003.

[9] J. H. He, "Limit cycle and bifurcation of nonlinear problems," Chaos, Solitons and Fractals, vol. 26, no. 3, pp. 827-833, 2005.

[10] J. H. He, "Application of homotopy perturbation method to nonlinear wave equations," Chaos, Solitons and Fractals, vol. 26, no. 3, pp. 695-700, 2005.

[11] J.-H. He, "Periodic solutions and bifurcations of delaydifferential equations," Physics Letters A, vol. 347, no. 4-6, pp. 228-230, 2005.

[12] J.-H. He, "The homotopy perturbation method nonlinear oscillators with discontinuities," Applied Mathematics and Computation, vol. 151, no. 1, pp. 287-292, 2004.

[13] J. H. He, "Homotopy perturbation method for bifurcation of nonlinear problems," International Journal of Nonlinear Sciences and Numerical Simulation, vol. 6, no. 2, pp. 207-208, 2005. 
[14] J.-H. He, "Asymptotic methods for solitary solutions and compactons," Abstract and Applied Analysis, vol. 2012, Article ID 916793, 130 pages, 2012.

[15] J.-H. He, "New interpretation of homotopy perturbation method," International Journal of Modern Physics B, vol. 20, no. 18, pp. 2561-2568, 2006.

[16] L. Cveticanin, "Homotopy-perturbation method for pure nonlinear differential equation," Chaos, Solitons and Fractals, vol. 30, no. 5, pp. 1221-1230, 2006.

[17] S. Abbasbandy, "Application of He's homotopy perturbation method for Laplace transform," Chaos, Solitons and Fractals, vol. 30, no. 5, pp. 1206-1212, 2006.

[18] M. Rafei and D. D. Ganji, "Explicit solutions of Helmholtz equation and fifth-order KdV equation using homotopy perturbation method," International Journal of Nonlinear Sciences and Numerical Simulation, vol. 7, no. 3, pp. 321-328, 2006.

[19] A. M. Siddiqui, R. Mahmood, and Q. K. Ghori, "Thin film flow of a third grade fluid on a moving belt by he's homotopy perturbation method," International Journal of Nonlinear Sciences and Numerical Simulation, vol. 7, no. 1, pp. 7-14, 2006.

[20] A. M. Siddiqui, M. Ahmed, and Q. K. Ghori, "Couette and poiseuille flows for non-newtonian fluids," International Journal of Nonlinear Sciences and Numerical Simulation, vol. 7, no. 1, pp. 15-26, 2006.

[21] J.-H. He, "Variational iteration method: a kind of non-linear analytical technique: some examples," International Journal of Non-Linear Mechanics, vol. 34, no. 4, pp. 699-708, 1999.

[22] J.-H. He, "Variational iteration method for autonomous ordinary differential systems," Applied Mathematics and Computation, vol. 114, no. 2-3, pp. 115-123, 2000.

[23] J.-H. He and X.-H. Wu, "Construction of solitary solution and compacton-like solution by variational iteration method," Chaos, Solitons \& Fractals, vol. 29, no. 1, pp. 108-113, 2006.

[24] J.-H. He, "Approximate analytical solution for seepage flow with fractional derivatives in porous media," Computer Methods in Applied Mechanics and Engineering, vol. 167, no. 1-2, pp. 57-68, 1998.

[25] J.-H. He, "Approximate solution of nonlinear differential equations with convolution product nonlinearities," Computer Methods in Applied Mechanics and Engineering, vol. 167, no. 1-2, pp. 69-73, 1998.

[26] J.-H. He, "Asymptotic methods: the next frontier towards nonlinear science," Computers \& Mathematics with Applications, vol. 61, no. 8, pp. 1907-1908, 2011.

[27] M. A. Abdou and A. A. Soliman, "Variational iteration method for solving Burger's and coupled Burger's equations," Journal of Computational and Applied Mathematics, vol. 181, no. 2, pp. 245251, 2005.

[28] A. A. Soliman, "A numerical simulation and explicit solutions of KdV-Burgers' and Lax's seventh-order KdV equations," Chaos, Solitons and Fractals, vol. 29, no. 2, pp. 294-302, 2006.

[29] E. M. Abulwafa, M. A. Abdou, and A. A. Mahmoud, "The solution of nonlinear coagulation problem with mass loss," Chaos, Solitons \& Fractals, vol. 29, no. 2, pp. 313-330, 2006.

[30] S. Momani and S. Abuasad, "Application of He's variational iteration method to Helmholtz equation," Chaos, Solitons \& Fractals, vol. 27, no. 5, pp. 1119-1123, 2006.

[31] N. Bildik and A. Konuralp, "The use of variational iteration method, differential transform method and adomian decomposition method for solving different types of nonlinear partial differential equations," International Journal of Nonlinear Sciences and Numerical Simulation, vol. 7, no. 1, pp. 65-70, 2006.
[32] Z. M. Odibat and S. Momani, "Application of variational iteration method to nonlinear differential equations of fractional order," International Journal of Nonlinear Sciences and Numerical Simulation, vol. 7, no. 1, pp. 27-34, 2006.

[33] J.-H. He, "Some asymptotic methods for strongly nonlinear equations," International Journal of Modern Physics B, vol. 20, no. 10, pp. 1141-1199, 2006.

[34] Z. Wan-Xie, "On precise integration method," Journal of Computer and Applied Mathematics, vol. 163, no. 1, pp. 59-78, 2004.

[35] D.-C. Wan and G.-W. Wei, "The study of quasi wavelets based numerical method applied to Burgers' equations," Applied Mathematics and Mechanics, vol. 21, no. 10, pp. 991-1001, 2000.

[36] G. W. Wei, "Quasi wavelets and quasi interpolating wavelets," Chemical Physics Letters, vol. 296, no. 3-4, pp. 253-258, 1998.

[37] S.-L. Mei, C. J. Du, and S. W. Zhang, "Asymptotic numerical method for multi-degree-of-freedom nonlinear dynamic systems," Chaos, Solitons and Fractals, vol. 35, no. 3, pp. 536-542, 2008. 


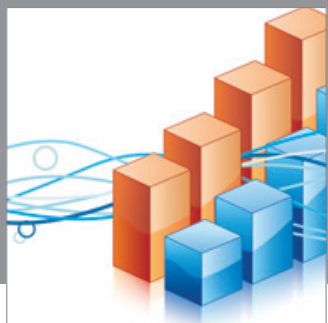

Advances in

Operations Research

mansans

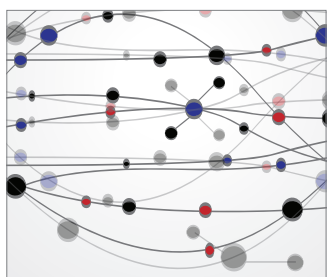

The Scientific World Journal
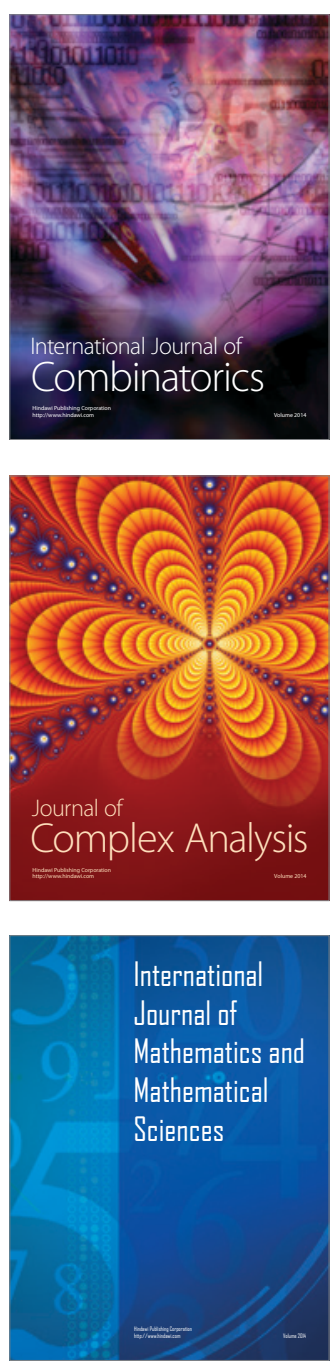
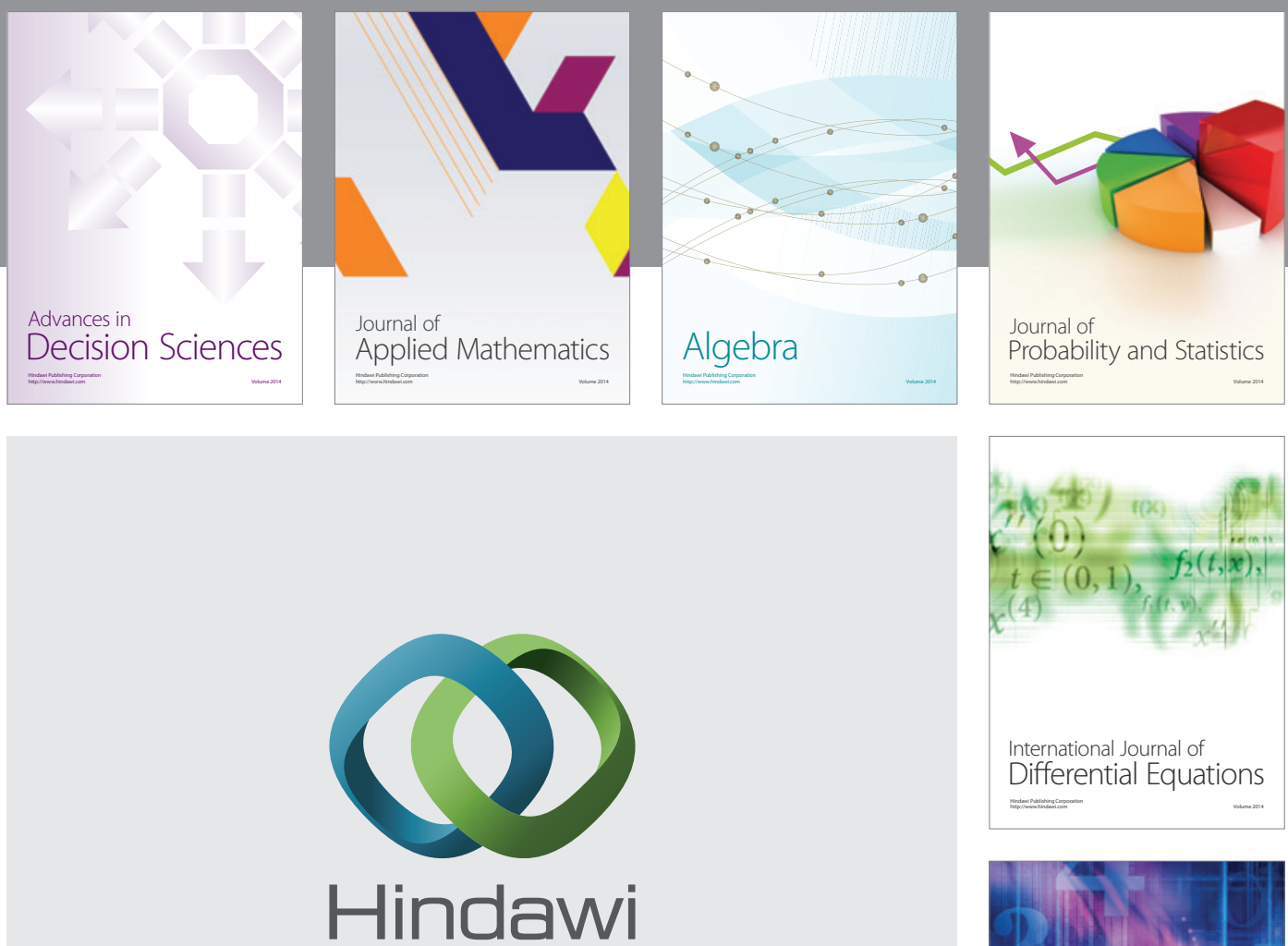

Submit your manuscripts at http://www.hindawi.com
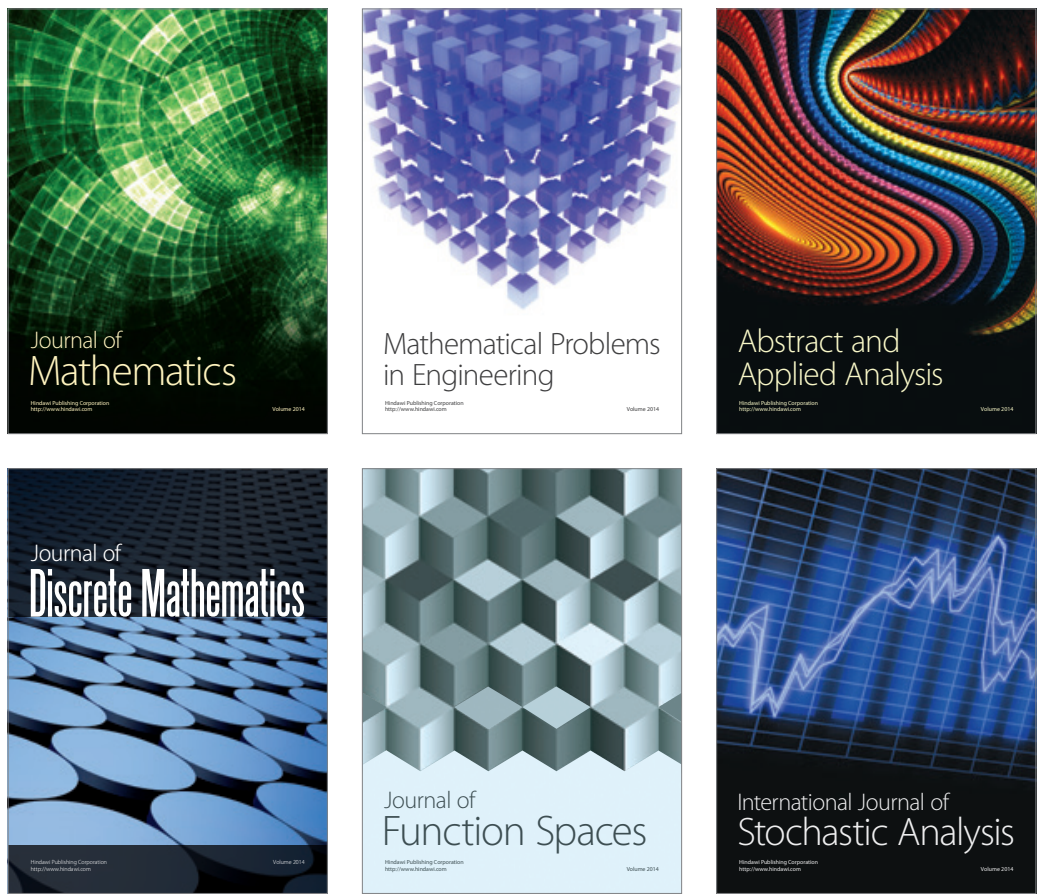

Journal of

Function Spaces

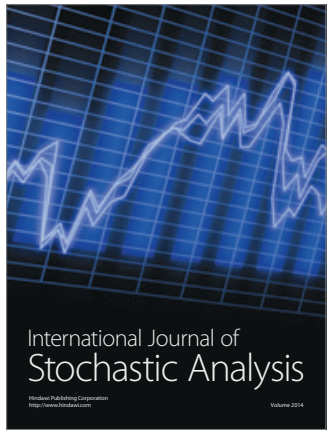

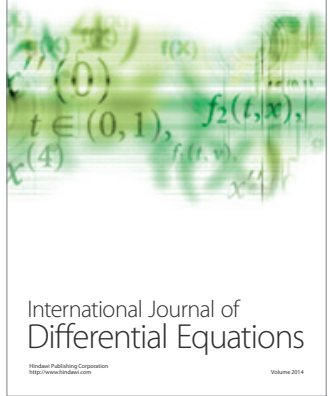
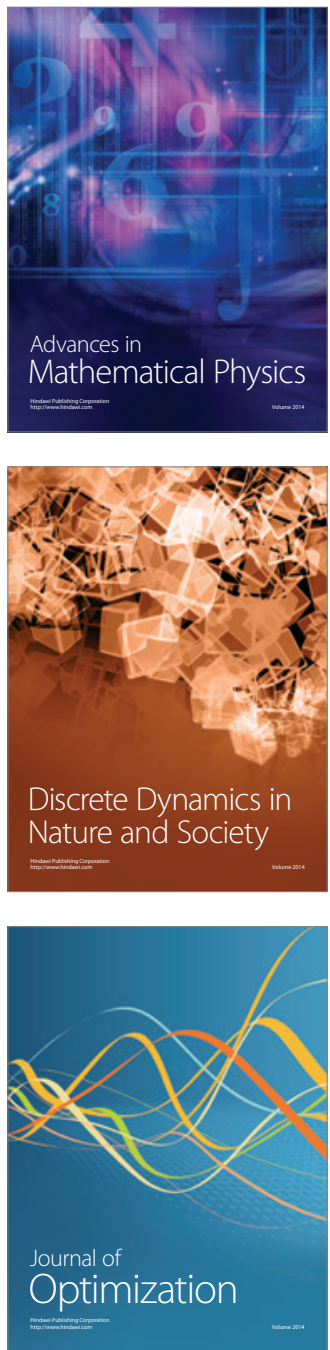